\title{
Correction: Amelioration of autism-like social deficits by targeting histone methyltransferases EHMT1/2 in Shank3-deficient mice
}

Zi-Jun Wang ${ }^{1} \cdot$ Ping Zhong ${ }^{1} \cdot$ Kaijie Ma ${ }^{1} \cdot \mathrm{Ji}^{-S e o n} \mathrm{Seo}^{2} \cdot$ Fengwei Yang $^{1} \cdot \mathrm{Zihua} \mathrm{Hu}^{3} \cdot$ Freddy Zhang $^{1} \cdot \mathrm{Lin} \mathrm{Lin}^{1} \cdot$ Jie Wang ${ }^{3} \cdot$ Tao Liu $^{4} \cdot$ Emmanuel Matas $\mathbb{D}^{1} \cdot$ Paul Greengard ${ }^{2} \cdot$ Zhen Yan $\mathbb{D}^{1}$

Published online: 13 September 2019

(c) Springer Nature Limited 2019

\section{Correction to: Molecular Psychiatry}

https://doi.org/10.1038/s41380-019-0351-2

published online 18 January 2019
Following publication of this article, the authors noticed that the Supplementary Materials and Methods file was accidentally omitted. This file has now been uploaded alongside the original article. This has been corrected in both the PDF and HTML versions of the article.

Zhen Yan

zhenyan@buffalo.edu

1 Department of Physiology and Biophysics, State University of New York at Buffalo, School of Medicine and Biomedical Sciences, Buffalo, NY 14203, USA

2 Laboratory of Molecular and Cellular Neuroscience, The Rockefeller University, New York, NY 10065, USA

3 Center for Computational Research, New York State Center of Excellence in Bioinformatics \& Life Sciences, State University of New York at Buffalo, Buffalo, NY 14203, USA

4 Department of Biochemistry, State University of New York at Buffalo, Buffalo, NY 14203, USA 